\title{
A Magnetic Nano-Particle Ink for Tunable Microwave Applications
}

\author{
Farhan A. Ghaffar*, Mohammad Vaseem \\ Electrical Engineering Program, CEMSE Division \\ King Abdullah University of Science and Technology \\ Thuwal, Saudi Arabia \\ farhan.ghaffar@kaust.edu.sa,mohammad.vaseem@kaust.edu.sa
}

\author{
Atif Shamim \\ Electrical Engineering Program, CEMSE Division \\ King Abdullah University of Science and Technology \\ Thuwal, Saudi Arabia \\ atif.shamim@kaust.edu.sa
}

\begin{abstract}
Inkjet printing or printing for realization of inexpensive and large area electronics has unearthed as an attractive fabrication technique. Though at present, mostly the metallic inks are printed on regular microwave substrates. In this paper, a fully printed multilayer fabrication process is demonstrated where the substrate is also realized through printing. A novel $\mathrm{Fe}_{2} \mathrm{O}_{3}$ based magnetic ink is used as a substrate while an in-house silver organo complex (SOC) ink is developed for metallic layers. Complete magnetostatic and microwave characterization of the ink is presented. At the end, a tunable patch antenna is shown as an application using the magnetic ink as the substrate. The antenna shows a tuning range of $12.5 \%$ for a magnetic field strength of $3 \mathrm{kOe}$.
\end{abstract}

Keywords—nano-particle ink, tunability, magnetic substrate

\section{INTRODUCTION}

Additive fabrication methods are gaining a lot of importance due to their low cost and ease of production. Generally fabrication techniques rely on subtraction which results in material wastage. As opposed to this, additive processes such as printing deposits material only where it is required hence reducing the material cost [1]. Moreover the roll-to-roll manufacturing capability of printing makes it a viable option for mass production in industries.

Passives such as antennas and inductors have been reported using printing for their realization [2]-[3]. Substrates such as Kapton and PEN are used to print metallic layers on them. Lately, printing of a polymer ink was shown in [4] to be used as a substrate as opposed to the usage of conventional microwave substrates. Silver ink was used for the deposition of metallic traces on the top of the polymer ink. In this paper, the authors propose the fabrication of magnetic ink to add functionality to the printed substrate which was shown in [4]. A fully printed process utilizing a custom ferrite $\left(\mathrm{Fe}_{2} \mathrm{O}_{3}\right)$ based magnetic ink and a silver-organo-complex (SOC) ink for metal traces printing is demonstrated. The magnetic ink is characterized for its low frequency and high frequency properties to study its viability in tunable microwave applications. It is known that applying the magnetic fields across a ferrite material varies its magnetostatic properties, which can be used for controlling the microwave performance of a component [5], [6]. As a proof-of-concept a tunability of a patch antenna around $8 \mathrm{GHz}$ is studied in this work. The results thus obtained shows that the magnetic ink is highly suitable for tunable/reconfigurable microwave devices.

\section{FABRICATION PROCESS}

The steps for the fabrication process are shown in Fig. 1. Due to the liquid nature of the magnetic ink, it requires a base material for its printing. For this purpose, FR-4 is used as a frame with a sacrificial paper at the bottom as shown in Fig. 1 (a). A slot $(24 \mathrm{~mm} \times 24 \mathrm{~mm})$ is created in the FR-4 frame where the ink is deposited. Using squeegee the magnetic ink is printed inside the slot. The ink is made up of $\mathrm{Fe}_{2} \mathrm{O}_{3}$ nanoparticles with SU8 epoxy resist in 50:50 weight \% ratios. Once the ink has been printed it is cured using ultraviolet rays for a period of $30 \mathrm{~min}$, Fig. 1 (b). With the help of the curing the ink solidifies and can be cut through and released from the holding substrate as shown in Fig. 1(c). It is worth mentioning here that at this point the magnetic substrate still has the support paper at its back which is removed by immersing it in warm water for few minutes. The use of manual squeegee as fabrication results in a rough the surface of the substrate which needs to be smoothen out. Therefore a smoothening layer of '3D vero black plus' is printed on the top of the printed substrate. At the end, SOC ink is deposited using inkjet printing, shown in Fig. 1 (d). A total of 8 layers of SOC ink are printed and cured using infrared (IR) heating for $5 \mathrm{~min}$.

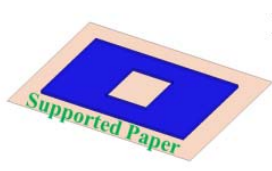

(a)

(d)

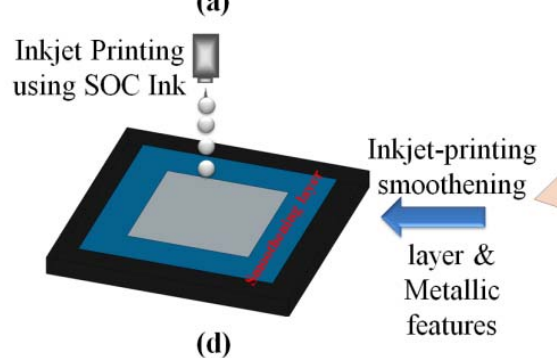

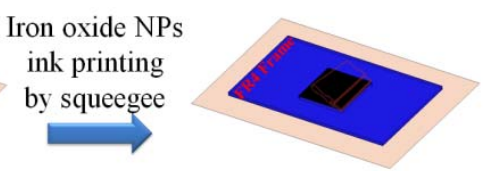

(b)

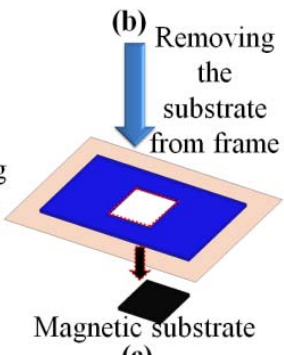

(c)
Fig. 1. Fabrication process 


\section{CharaCteriZATION OF MAGNETIC INK AND ANTENNA}

\section{A. Magnetostatic and Microwave Properties of the $\mathrm{Fe}_{2} \mathrm{O}_{3} \mathrm{Ink}$}

To use a magnetic substrate for microwave design, it needs to be characterized for its magnetostatic and microwave properties. Therefore, the $B(\mathrm{H})$ curve of the printed magnetic substrate is extracted using Vibrating Sample Magnetometer (VSM). A saturation magnetization $\left(4 \pi M_{\mathrm{s}}\right)$, coercive field $\left(H_{\mathrm{c}}\right)$ and remanent magnetization $\left(B_{\mathrm{m}}\right)$ of $1560 \mathrm{G}, 46 \mathrm{Oe}$ and $350 \mathrm{G}$ respectively are obtained from the measurement. The most critical among these properties for a microwave design is the saturation magnetization which determines the magnetization frequency $\left(f_{\mathrm{m}}=\gamma 4 \pi M_{\mathrm{s}}=4.37 \mathrm{GHz}\right)$ of the substrate beyond which the substrate has low loss behavior.

A ring resonator is designed on the substrate for its microwave characterization. Although the resonator is measured from $0.5 \mathrm{GHz}$ to $10 \mathrm{GHz}$, the properties beyond 4 $\mathrm{GHz}$ are of interest as explained above. Effective dielectric constant of 13, 12 and 15 are obtained at $4 \mathrm{GHz}, 6 \mathrm{GHz}$ and 8 $\mathrm{GHz}$ respectively. The high dielectric constant value is expected due to the ferromagnetic nature of the ink. The loss tangent value is around 0.01 which is typical for such substrates. These results are shown in Fig. 2 (a).

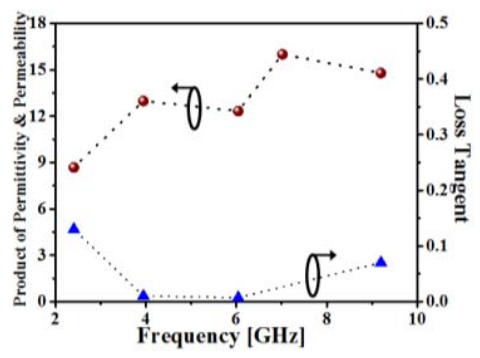

(a)

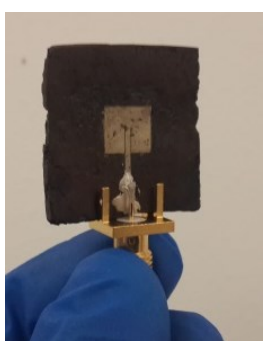

(b)
Fig. 2 (a) Dielectric and magnetic properties of magnetic ink (b) Fabricated patch antenna

\section{B. Patch Antenna Design}

After the ink has been characterized for its low and high frequency properties it is now used for demonstration of an application. A linear polarized patch antenna operating at 8 $\mathrm{GHz}$ is used as shown in Fig. 2 (b). This frequency is selected to stay away from the low field loss region which is below 4 $\mathrm{GHz}$. The antenna has dimensions of $6.4 \mathrm{~mm} \times 7 \mathrm{~mm}$. At first the antenna is measured for its impedance and radiation performance without any bias. A maximum gain of $-0.7 \mathrm{dBi}$ is achieved at $8.2 \mathrm{GHz}$. The inefficient gain value of the antenna is due to the surface roughness of the printed metal. A measured roughness value of $4.5 \mu \mathrm{m}$ is observed from the Profilometer. Without this roughness, the gain of the antenna is around $3 \mathrm{dBi}$ in simulations which is typical for ferrite based antennas. However, when the antenna is simulated with the measured metal surface roughness this value drops to -0.5 $\mathrm{dBi}$. These results indicate that if the roughness of the fabricated metal can be improved, it can result in much better gain value.

The impedance and radiation properties of the antenna in the unbiased condition are acceptable for tuning measurements. To accomplish this, a magnetostatic field is applied across the antenna using an external electromagnet. The magnetic field strength is varied from 0 Oe to $5 \mathrm{kOe}$. Initially no change is observed in the center frequency of the antenna up to a bias strength of $2 \mathrm{kOe}$. As soon as the field intensity is increased beyond $2 \mathrm{kOe}$, the resonant frequency of the antenna starts to tune down as shown in Fig. 3 (a). From 2 $\mathrm{kOe}$ to $3 \mathrm{kOe}$ the antenna shows a tuning range of $1.25 \mathrm{GHz}$. This range is almost $12.5 \%$ of the center frequency. Further increasing the bias shows that there is a slight increase in the resonant frequency of the antenna. This can be due to the fact that the substrate is saturated for the bias field strength of 3 kOe. This means that the properties of the material are now governed by Polder's equations. The reflection coefficients of the antenna in the unbiased and biased states are shown in Fig. 3 (b). No significant effect is expected on the antenna radiation pattern due to the applied bias as it has been reported previously that the radiation pattern of ferrite based patch antenna does not change much in the biased state [6].

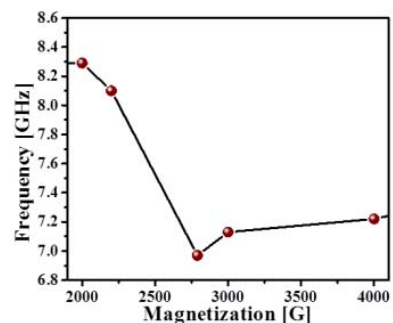

(a)

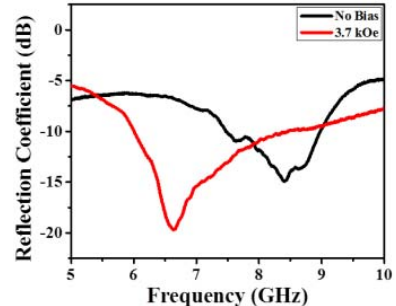

(b)
Fig. 3 (a) Measured frequency tuning (b) $S_{11}$ measurements for no bias and $3.7 \mathrm{kOe}$ bias

\section{CONCLUSION}

Fabrication and characterization of a fully printed $\mathrm{Fe}_{2} \mathrm{O}_{3}$ based magnetic ink is presented in this paper. A simple patch antenna is designed and measured to prove the viability of the ink as a substrate for tunable microwave components. The antenna exhibits a tuning range of $12.5 \%$ around the center frequency of $8 \mathrm{GHz}$ for a magnetic field strength of $3 \mathrm{kOe}$. The functionality of the ink demonstrates that it can be used for tunable and reconfigurable microwave applications.

\section{REFERENCES}

[1] J. G. Korvink, et. al., "Inkjet-based micro manufacturing", Weinheim: Wiley-VCH, 2012.

[2] M. Vaseem, et.al, "Robust Design of a Particle-Free Silver-OrganoComplex Ink with High Conductivity and Inkjet Stability for Flexible Electronics", ACS Applied Materials \& Interfaces, vol. 8, no. 1 pp. 177186, 2016.

[3] M. M. Khan, et. al., "3.56-bits $/ \mathrm{cm}^{2}$ Compact Inkjet Printed and Application Specific Chipless RFID Tag," in IEEE Antennas and Wireless Propagation Letters, vol. 15, pp. 1109-1112, 2016.

[4] G. McKerricher, et. al., "Fully Inkjet Printed RF Inductors and Capacitors using Polymer Dielectric and Silver Conductive Ink with Through Vias", IEEE Transactions on Electron Devices (TED), vol.62, no.3, pp.1002-1009, 2015.

[5] F. A. Ghaffar, et. al., "A Ferrite LTCC Based Dual Purpose Helical Antenna Providing Bias for Tunability," IEEE Antennas and Wireless Propagation Letters, vol. 14, pp. 831-834, 2015.

[6] F. A. Ghaffar, et. al, "Theory and design of a tunable antenna on a partially magnetized ferrite LTCC substrate", IEEE Transactions on Antennas and Propagation, vol. 62, no. 3, pp. 1238-1245, 2014. 